\title{
Managing Costs and Risks
}

\begin{abstract}
This chapter identifies some of the 'costs' associated with health worker placements in low-resource settings; it begins with a discussion of the costs of covering staff time during placements and some of the fears surrounding skills 'wastage' before highlighting risk areas associated with such placements.
\end{abstract}

Keywords Costs $\cdot$ Risks $\cdot$ Skills wastage $\cdot$ Measurement

\section{Background: the NHS in Fiscal Crisis?}

On the basis of the research we have conducted and our experiential learning as managers of professional volunteering projects, we have no doubt that placements in low-resource settings both identify and invest in entrepreneurial knowledge and innovation. We also know that the remodelling of care in the current NHS environment in response to rising levels of chronic and complex conditions requires new forms of critical connective knowledge. Addicott et al. (2015) express the need for, 'closer attention to the role of generalists [in an environment] where care is delivered in teams based around the patient rather than in professional silos' (2015: 34). International placements deliver precisely this form of knowledge. But this learning, however valuable in its own right, is taking place in a specific economic, political and temporal context. The NHS is facing a financial crisis. And this is best characterised as a human resource crisis, as $70 \%$ of 
recurring costs relate to staffing (Addicott et al. 2015: 2). The 'crisis' is specifically manifest in an alarming growth in reliance upon temporary and agency staff. According to a recent document, NHS spending on agency staff has 'increased to the extent that it is one of the most significant causes of deteriorating Trust finances' (NHS Improvement Agency Rules 2016: 4). New rules designed to control this spend have introduced a cap (ceiling) on the level of pay that NHS employers can offer agency staff; in the case of junior doctors and other medical/clinical staff the cap is set at $55 \%$ above basic pay rates. The document explains how to cost agency staff using specified hourly rates and provides illustrative costs for a consultant doctor at $£ 652.96$ for an eight-hour shift. Based on these figures, providing staff cover or 'back-fill' for a junior doctor on an international placement for 12 months could cost around $£ 66,760$ (p. 20). ${ }^{l}$ The Royal College of Nursing also produced a report on the 'unprecedented' reliance on agency nurses in the current 'perilous' financial crisis and projected an annual NHS spend of at least $£ 980$ million on agency nursing by the end of 2015 (RCN 2015: 3 ). This is the environment within which the MOVE project was tasked to weigh up the costs and benefits associated with professional volunteering.

The costs identified above concern only replacement salaries. What if the placements themselves generate new costs in relation to the individuals involved? Sherraden et al. (2008) suggest that the overwhelming majority of studies focus on the positive returns on professional volunteering with little attention paid to costs. In some ways, even personal experiences which are ostensibly (or actually) negative at the time can be regarded as having a positive effect in the long run. As we have noted, experiencing failure can stimulate innovation. However, there are, largely unsubstantiated, concerns that long periods overseas can result in elements of skills wastage, absorption within cultures of bad practice or desensitisation to risk. More specifically, there is a genuine risk that personnel may be exposed to infectious disease, terrorism, road traffic accidents and other forms of stress or trauma which generate new costs for the NHS. The systematic review conducted as part of the MOVE project identified a number of perceived 'externality' effects. These can be loosely grouped as follows:

1. Financial costs of staff replacement (as above)

2. Potential career risks (re-integration, negative attitudes of colleagues, professional revalidation)

3. Risk components (insurance, health and safety and security issues, personal trauma). 
4. Cultural awareness

5. Personal impacts (loneliness, relationship tensions, fears of becoming judgemental on return)

6. Environmental impacts associated with air travel.

These concerns map onto the findings from the interviews with returned volunteers, although it must be said that the balance tips very strongly in the direction of benefits and these issues are typically mentioned only when respondents are encouraged to think in those terms. The following section discusses some of the issues raised.

\section{Professional Voluntarism and 'Brain Drain'}

The most tangible and immediate risk to the NHS is for the placement experience to stimulate a desire for future mobility or eventual emigration. It is well established that early career or early life mobility tends to foster a desire or the confidence to engage in future mobilities (Ackers 2003; King and Ruiz-Gelices 2003). We have also identified a number of cases where individuals were motivated in the first instance to engage in professional voluntarism as an active decision to develop a career in global health. The following case is illustrative:

I had an ulterior motive, I applied for MSF (Médecins Sans Frontières) a while ago and I didn't have enough management skills. I've worked in the humanitarian sector before but not with front line stuff.

The line manager in the following case suggests that some volunteers may find it difficult to return to the NHS and take the decision to engage in further international work once they return. She does suggest that it may not be the placement as such but the personality involved that precipitates this more 'footloose' approach to career planning:

I think it is hard coz sometimes when they come back I think they don't fit here anymore and they leave again. Those with certain types of personality probably would have - its gonna happen anyway, but maybe it happens earlier [Line Manager]

In this and several other cases, the applicant was already planning to leave the NHS, at least temporarily, for a career in a low-resource setting or in 
global health more generally. In such situations, international placements provide important opportunities to take the first steps for leaving the NHS. In one case, the volunteer later signed up for a master's programme in global health; in another, a midwife left her position in the UK prior to departure with no intention to return immediately after her placement. Of course, these plans subsequently change and individuals may actually return to the NHS either immediately or in the future. In one case, a volunteer who took up a placement immediately after completion of a master's programme in global health and actively planned a career in global health at that stage in their career later returned to the UK to commence nurse training. Further down the line, he may elect to spend some time in a nursing capacity in a low-resource setting.

Having said that, in most cases where mobility creates an appetite for future work in low-resource settings this takes the form of an interest, often transformed into action, in getting involved in subsequent short stays (typically two weeks taken during vacations or study leave) perhaps to deliver specific training or 'virtual' engagement through mentoring of colleagues. The opportunities for individuals undertaking placements in low-resource settings to up and leave the NHS are very limited and restricted to those specifically interested in global health. On that basis, there is far less risk that they will leave to take more attractive positions or transition into long-term or settled forms of migration as the labour markets are simply not conducive to that. It would be rare to find professional employment in a low-resource setting that could even begin to sustain an employee let alone a family. In that respect, international placements in high-resource settings pose a much greater 'brain drain' risk. Recent interviews with British doctors and nurses in New Zealand identified a strong relationship between early career placements and future career decisionmaking facilitating the longer-term emigration of UK junior doctors. ${ }^{2}$

Career decision-making is increasingly boundaryless (Arthur 1994, 2014; Arthur and Rousseau 1996) and less likely to follow simple linear pathways and individuals change their perspectives over time. Whilst placement experiences may provide an opportunity to test the waters and potentially leave the NHS, they may also provide the impetus for a decision to return to it with renewed motivation:

I was getting frustrated with the NHS cos I couldn't get away to volunteer. My Deanery were like, sorry [name]. Where I wanted to go wasn't recognised as a training placement as there were no senior medics there. I was 
seeing colleagues who were going away for what was effectively a year in Australia and places like that. It's supposed to be training, but half the time it's sitting on Bondi Beach. Or they were doing management fellowships where they sit in an office for a year. But I was feeling like I wanted to do something that was actually useful to other people. I got very frustrated, so I decided I needed to resign and once I'd decided, it all sort of fell into place. I'm now back in the NHS, working as a locum registrar, but my intention is to get back into the NHS as a permanent registrar. I think I'm more committed now than before I left, but if I hadn't have left when I did, I might not have come back.

\section{Skills Wastage}

The next issue raised with some regularity (but once again by a minority) concerns potential skills wastage. There is no hard evidence in existing research or in our own empirical work of actual skills waste on return. However, prior to return, many volunteers do experience some anxiety about their ability to slot back into the NHS environment with its rigid protocols, hierarchical structures and high-tech equipment. Once they have returned and reintegrated into the NHS workforce, they typically reflect on this as a momentary confidence issue due to the gap in their use of specific types of equipment. This concern links nicely to the ideas of deliberative practice discussed earlier suggesting that anyone who is not regularly using a skill may experience some decline in confidence. Anaesthetists were most likely to raise this issue:

I'm definitely de-skilled in the high-tech side of things, which is what I expected beforehand. All this high-tech equipment like fibre optic intubations. Even Epidurals. But that doesn't worry me because once you get back into your normal practice we quickly pick that up. At home working fulltime two or three weeks you'll be back to normal.

The first comment, 'I'm definitely de-skilled' is a little alarming but is immediately followed by the reassurance that this de-skilling is very short lived and may be more about confidence and perception than explicit skills. In another case, a midwife expressed some anxiety about water births:

I've gone back to post-natal first, so I'm not going straight back into labour suite yet. I'm scared of water births strangely, but I know if I call for help it will be there. 
In other cases, respondents talked about doing some prior reading to prepare themselves, perhaps building on research skills and a 'can do' attitude honed during their placement:

There are things I will have to go back and read up like patient monitoring we would never use that here because it's just too modern, but I think it will just take a day or so.

Chapter 3 discussed the benefits of multi-professional working and working at the boundaries of one's own profession. This was viewed as an important benefit by most volunteers and a key locus of new learning. However, a small number of volunteers also expressed some concerns about the impact of this on their subject-specific knowledge. In one case, a nurse who had been working in paediatrics in the UK applied for a placement involving work with neonates. Although she enjoyed the challenge of working in this rather new environment on a neonatal unit, she did begin to express a desire to work with older children which fell outside the scope of the project and articulated this in terms of a decline in her specialist skills:

This was always going to be a bit of a challenge because I've not worked with neonates before and I feel like we're [now] going in more of a midwifery direction. I'm actually quite happy to do that for a week or so but I wouldn't fancy for long term.

The study also identified isolated perceptions of what can best be described as a lapse into bad practice or 'cutting corners'; interestingly these are always reported 'second-hand' about a third party. One volunteer described a situation she had observed where a nurse who had been away from the UK for two years (deployed through another organisation) had begun to adopt some of the bad practices of local staff in relation to routine patient monitoring and recording. Another British nurse expressed concern that her volunteer colleagues were lapsing into bad practice rather than encouraging best practice amongst their peers:

It's ended up that even the white people are doing that now. You were talking about de-skilling - what we're doing is changing our ways to cope with theirs, to fit in with them and suit theirs. We're seeing more of that from the white people rather than the other way around. 
This respondent was one of very few cases of volunteers who requested to return to the UK prematurely after four months of a six-month placement. She had found leaving her teenage children, as a single parent, very challenging and found it hard to cope with the level of what she saw as negligence on an overwhelmingly busy maternity unit. It is difficult to know whether the practices she refers to concern professional volunteers making some necessary adjustments to their working practices to meet the needs of the resource-constrained local environment and 'make do' but without any de-skilling taking place as such, or whether they were simply taking the easy option and losing their sense of professionalism. Our observational research would certainly indicate the former and suggest that there is little risk that staff would not adhere to protocols on their return, although many did not relish a return to the level of bureaucracy and form-filling associated with the NHS. The following doctor explains how he had to 'make do' but this represented a conscious and necessary response to the situation and not a preference or indication of laziness:

In this country we're highly specialised and we have to learn how to use a lot of equipment. When people go over there that's stripped away and you're just working with the bare bones. It's your responsibility to make do with what you've got, and that's an eye opener for people but I don't think people like to work that way. They like the security of working within the NHS and having skilled people around them. It's a lot safer.

In the following case, an anaesthetist had only allowed herself one week on her return home to relocate to a new position. This situation is quite common amongst junior doctors who rotate annually and will be commencing an entirely new position on return. Nurses and midwives more usually return to their old position reducing overall dislocation. It is interesting to see how this doctor talks about her communication skills:

How are you feeling about returning to the NHS?

Nervous, I haven't got any downtime between coming back and starting my job. I'm working in a brand new city, a brand new hospital, new doctors and I don't have a car or a house. I only have a week from arriving home to starting my job. I feel like I'm out of practice in a lot of things; I haven't done any gynaecology out here, I'm out of sync with all the good normal 
practice that we should be using. Even communicating, I had a friend come out to stay and she asked me why I was talking in a ridiculous voice; she said I was talking really slowly. You realise you adopt a way of speaking so people understand you better, so to go back it's going to be really hard to kick out of it and speak normally.

There was some concern that extended stays could result in disengagement with the latest evidence-based practice and technological advances. In the following case, the respondent refers to an American colleague who has organised periods of repatriation to enable her to keep up to the speed of the latest research and advances:

One of the clinicians from the [US] group is going to be there for three to five years. Obviously that is going to have a huge impact on her clinical practice where you cannot do basic investigations and things are moving on, there are papers being published every week that she is missing out on so they have set it up for her that every three or four months she goes back and gets updated.

This may well be a matter of confidence and clinical practice rather than knowledge as such as most volunteers will be able to access published papers and gain quite significant research skills during placements. Indeed, from an ethnographic perspective, the volunteer house in Kampala had an atmosphere of research - of constantly discussing and researching conditions and potential interventions. This discussion took place on a 24/7 basis so much so that visitors and on rare occasions volunteers themselves had to extricate themselves to gain some reprieve.

The interviews, unsurprisingly, suggested a close relationship between the loss of confidence and length of stay. Longer stays tend to be associated with a greater decline in confidence in certain skills. On the other hand, this has to be balanced with the potential for skills gains in other areas which may derive from longer stays and, importantly, as this respondent points out, the relative benefits of the host setting:

If you're coming for a month it's not worth it; you're only going to take. You won't be able to give that much in the space of a month - I've learnt and got so much out of it and really enjoyed it and just thought ... the time has flown and that's the sign of a really positive experience. If people wanted to really do a lot of work I would struggle not to say, 'go for six months minimum.. a year or even better, two? 
Do you think the longer the better?

I do but there is a thing in that if you're out of the UK working or the US or the Western world of medicine are you deskilling yourself and are you as up to date and providing as good an obstetric advice as you could be? I've only been out four months, but I'm when I go back, I have a week of working with another midwife reorienting myself because I'm so out of the practice with writing notes and doing what we consider to be really top obstetric care... I need to re-familiarise myself with the speed at which I have to work at so I think if you're out for longer than a year you might lose a few skills...

One volunteer was faced with limited career options on return as her line managers had assumed a level of deskilling. Without any explicit consultation or communication, the decision was made to require her to work in the teaching hospital on her next rotation. At the time of interview this doctor was a little perplexed at the attitude of her employers and their implicit assumption that she posed something of risk especially as she had prepared herself for precisely that situation:

I didn't do foetal monitoring there. I think that is why they were concerned about me coming back so before I came back I made sure I refreshed my skills and stuff.

Asked whether she felt she had suffered from skills wastage and this had affected her practice on return, she replied: 'For me no, because I just think Uganda is different to the UK, I just adapt. You work to your environment. I just know it's a different system.'

An optional period of reintegration was subsequently built into the SVP program allowing one month on return when the volunteer could continue to receive their stipend and work on an unpaid basis. Experienced clinicians linked to the health partnerships and based in UK hospitals actively offered to mentor returned volunteers during this period. In practice, not one volunteer took up the offer.

In Chapter 3, we referred to the distinction between explicit and tacit skills and the growing emphasis on what can perhaps best be called 'qualities' or professional attributes rather than skills. Tolerance is frequently cited as an example of attitudinal change brought about through placements in low-resource settings. However, respondents on some (relatively few) occasions suggested that their experiences of working in resource-constrained environments and witnessing the stoicism of these 
patients may make them less tolerant of the more privileged patients in the UK. An experienced nurse with extensive experience in low-income settings highlighted an issue that many of our respondents mentioned:

[when I came back] I had to be careful that I didn't judge people too much who chuntered about petty things. Because, you know, these complaints that patients and staff will make... I just stood there and I just thought, I really wanted to say this really isn't anything for you to complain about. You know, you want to be where I've just been and seen what I've seen.

\section{EXPOSURE TO Risk AND Vicarious LiabiLITY}

Risk is a major driver of policy and practice in large organisations such as the UK National Health Service. The systematic review identified a number of potentially negative impacts of international placements linked to risk, including insurance, litigation, health and safety, and security issues and personal trauma. As managers, our experience of managing and evaluating risk responsible for volunteer deployment, and more recently, the Ethical Electives Project, has emphasised the importance of distinguishing genuine and tangible risks from perceptions of risk. International placements in low-resource settings expose individuals and organisations to risk. Inflated, generalised or misguided perceptions of risks amongst potential volunteers, their families, line managers and policy makers add a further dimension generating obstacles to mobility (Ackers et al. 2014). Aversion to risk may damage learning and reduce opportunities for innovation.

The SVP commissioned a professional risk assessment undertaken by the Chief Risk Officer at the University Hospital of South Manchester NHS Foundation Trust (Paul Moore). In addition to his background in risk management within the NHS, Moore had also completed risk assessment on the ground in Uganda for the Man-Gulu Health Partnership. The risk assessment report describes risk management proactively as a process of anticipating the effect of uncertainty on the achievement of programme objectives and building resilience to mitigate that uncertainty (Moore et al. 2015). It distinguishes 'inherent' from 'residual' risk which is defined as 'the estimated level of risk exposure after taking additional steps to control (or mitigate) risk' (p. 8). Figure 4.1 presents the inherent risks identified, and Fig. 4.2 identifies the residual risks at the time of the 


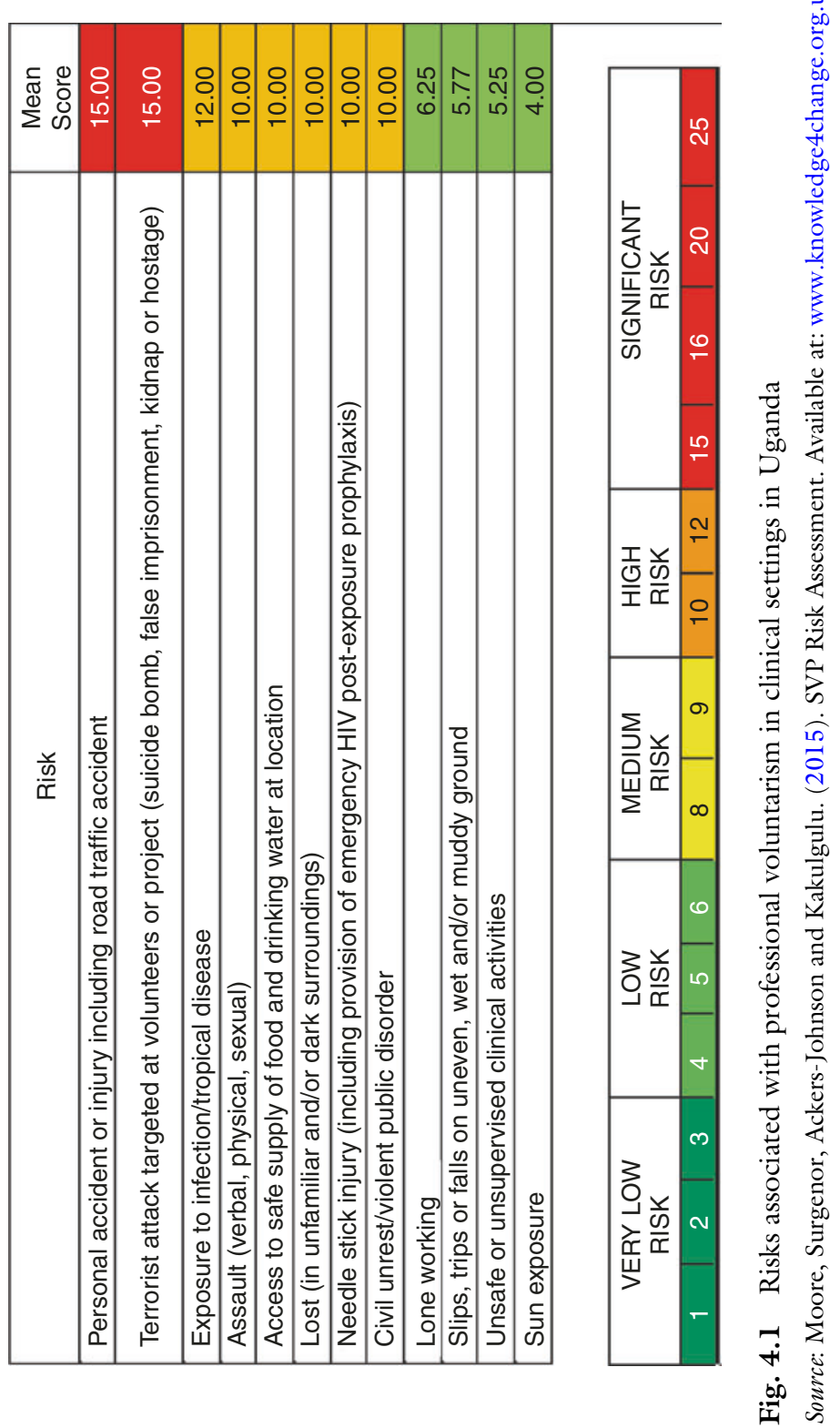




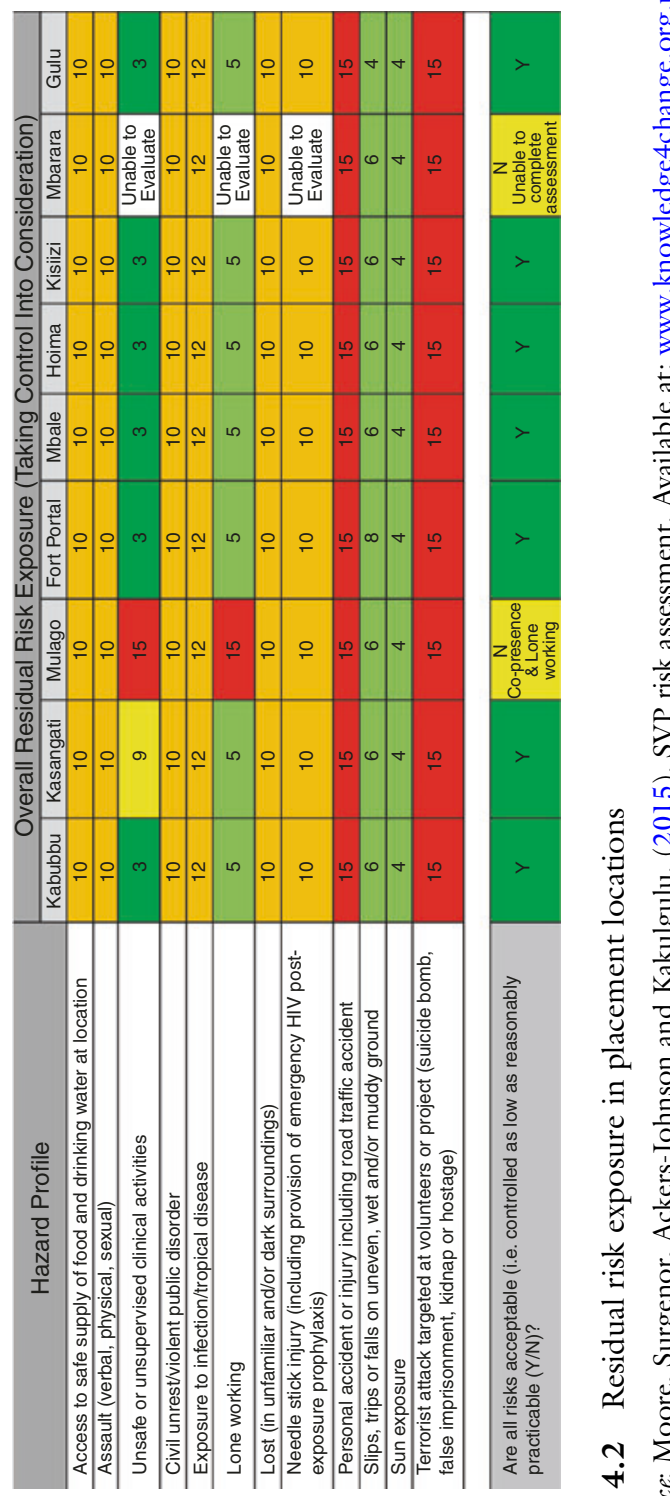

ํㅡㄹ

bै

i

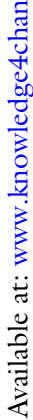

过

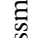

$\ddot{\infty}$

菂

s

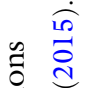

艺

氖

2.

$\Xi$ 일

긍

它

萂

芩

?

¿

×

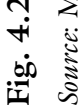


initial risk assessment visit that was conducted prior to project commencement:

The judgement about whether identified risks are 'acceptable' or not in a particular context requires the relative balancing of risk 'severity' with risk 'likelihood.' On that basis risks associated with road traffic accidents or terrorism are graded as severe, although their likelihood may be low and mitigated by effective behaviour management. This process stimulated and informed the development of SVP volunteer management systems largely reflected in an evolving induction process and associated induction pack. ${ }^{3}$ Over the past five years, the SVP has encountered most of the risks identified above. At the more alarming and less 'likely' end of the spectrum, there have been outbreaks of terrorism and civil disobedience (typically quite isolated and around election periods or involving tribal disputes). Road traffic accidents remain one of the greatest risks to volunteers in all low-resource settings (Gedde et al. 2011: 186). Bhatta et al. (2009) confirm the importance of road traffic accidents as a major cause of volunteer morbidity and mortality. In a survey of VSO volunteers, diarrhoea was the most prevalent heath risk reported (by 79.9\%) with highest levels found amongst short stays and younger volunteers. This is followed by skin and dental problems and $17.5 \%$ reported some form of road traffic injury. The authors are clear to point out that the response rate to their survey was small (36\%) and that this response rate may be skewed in favour of those who had experienced problems (and had something interesting to report). The situation is exacerbated in the Ugandan context by the use of 'boda bodas' (motorcycle taxis) and a study of Peace Corps volunteers in Africa found that $60 \%$ of road accidents were related to motorcycle use (Bernard et al. 1989). These risks to individuals also present a risk to the NHS potentially affecting the subsequent safe return of work-fit employees.

Exposure to infectious disease presents more direct potential impacts on return and these are risks that the wider public may become aware of and sensitised to. ${ }^{4}$ Since the commencement of the SVP, Uganda has experienced at least three localised outbreaks of Ebola and Marburg haemorrhagic fever. We have also witnessed several instances of needlestick injury. Both of these were responded to immediately triggering expert advice and a constant iteration of the induction pack.

Whilst NHS employers may be interested in the well-being of employees on placement, they are also anxious about the liabilities associated with that and the impact that any local incidents may have on volunteer's fitness 
to practice on return. This raises issues around clinical registration on placement and professional indemnity insurance.

All of these risks can be mitigated to some extent through attentive volunteer management supported by active relationships on the ground. It may provide some reassurance to readers to point out that over the past five years we have deployed well over 60 long-term professional volunteers and 110 undergraduate students in Uganda without any serious adverse outcomes.

One area highlighted in the risk assessment that is often overlooked and compounds all of the risks referred to above is that of lone or unsupervised working. We have discussed some of the implications of supervision in relation to optimal learning outcomes (Chapter 3). In some situations it could be argued that lone working or working without close supervision generates profound opportunities for innovative learning especially for more senior volunteers. International placements certainly attract individual clinicians keen to work outside the perceived constraints of NHS structures. The risk assessor described volunteering in low-resource settings as a 'magnet for mavericks' during initial discussions about risk and this is certainly confirmed by our experiences of recruiting and subsequently managing volunteers, especially doctors and more senior doctors some of whom actively seek out opportunities for what one junior doctor termed 'ninja medicine'.

Managing volunteer-health worker relationships has formed a major focus of the SVP process leading to the operationalisation of rigorous systems to prevent lone working for all cadres of staff. Drawing on previous research on highly skilled migration and knowledge mobilisation we have imported and operationalised the concept of 'co-presence. ${ }^{, 5} \mathrm{Co}$-presence is a necessary (but not sufficient) condition for mutual learning and reduces the risks associated with gap-filling and labour substitution (Ackers and Ackers-Johnson 2014). It is also a key component of risk mitigation. At one level, it links to other health risks, such as needle stick injury where policy requires that the individual must seek immediate HIV prophylaxis. It is also a key component of managing litigation or more commonly in many low-resource settings, the impact of blame cultures. In this environment, having witnesses is of critical importance. At another, more complex level, it requires and assists professional volunteers to manage the daily pressures on them to perform out with their competency.

Many medical and nursing students and junior doctors in low-resource settings are required to work without supervision often running services 
on their own (Ackers et al. 2016). This environment puts huge pressure on UK volunteers to do likewise. This 'expectation' and peer pressure is further compounded by irresponsible international organisations that place volunteers in gap-filling roles without adequate supervision or support. The risk assessment visit identified a shocking example of this, involving British medical students encouraged by their 'virtual' UK clinical supervisor to work at nights for data collection purposes:

Medical students explained how they were often goaded into carrying out clinical examination or diagnostic procedures they did not feel competent to perform, and whilst they declined to carry out the procedures, they explained how this created some tension with Ugandan medical students also working at the Hospital (Moore et al. 2015: 15)

Concerns about lone working and the complexities of establishing rules that were suitable to a wide range of volunteers from different professions, levels of seniority and personal competence/confidence led to the codevelopment of the 'SVP Competency Algorithm.' This was developed as a flexible working guide to empower volunteer decision-making when confronted with lone working or lack of effective supervision.

The case cited above involved students and UK volunteers who were working at night. The risks associated with lone working are elevated during night time and weekend working. A simple response to this is to prevent volunteers from working during these times and ensure that local staff are aware that this 'rule' is imposed by the deploying organisation rather than a simple volunteer preference. The issue of working hours is also present in some volunteer interviews. In keeping with the spirit of UK law the SVP instituted strict policies on working hours to prevent over working and the risks of exhaustion and trauma arising from that. The general lack of adherence to timetables in Uganda and many other lowresource settings can place pressure on those staff who are committed to their work to work extremely long hours to cover for absenteeism and poor time management. In most cases it is concerns about patients and leaving wards unattended that encourages long working hours. However, some volunteers based in mission hospital facilities faced undue pressure from British managers to commit to arduous working schedules that would not be permitted in the UK and subjected to humiliation if they questioned this. Weekly reporting mechanisms and close relationships with volunteer managers enabled us to support volunteers in these 
circumstances and to relocate volunteer to other Health Partnerships if situations did not improve. ${ }^{7}$

The research has identified a number of other areas of concern which have the potential to cause stress to professional volunteers. In many ways these sit hand-in-hand with key areas of learning underlining our contention that some of the most acute learning arises from discomfort. Whilst positive learning may indeed accrue, it is important that professional volunteers are protected and supported. The first of the areas concerns experiences of overt discrimination. The most prevalent form of discrimination identified involves attitudes towards women. A mid-career anaesthetist describes her experience:

I have come up against fairly misogynistic attitudes and several misunderstandings about whether or not I am actually a doctor. In fact, I am fairly certain on one occasion my ability or qualifications for teaching on the neo natal resuscitation course were questioned.

The following volunteer had six years specialist experience:

People here think that I am younger than I am definitely, but my personal perception of the relationship that I have with [in-charge doctor] is that he does not take me particularly seriously. I thought maybe that was gender. I constantly had to prove myself but at the same time when the department had no doctors he was more than happy for me to go on the rota [alone] and do ward rounds.

Gender emerges with some regularity in volunteer narratives in the SVP. This reflects the feminised nature of employment in maternal health and the stronger tendency for women to volunteer (Hudson and Inkson 2006: Bhatta et al. 2009). The strongly hierarchical nature of professions and organisations in many low-resource settings generates a high potential for gender-based discrimination. This should not be confused with the more composite concept of 'culture/religion' that often conveniently obscures gender/human rights dynamics. The SVP project with its emphasis on maternal and new-born health was strongly skewed in favour of female volunteers (Table 4.1).

Most of the female doctors deployed within the frame of the SVP reported experiencing patronising behaviour from senior peers and managers. In many cases this manifests itself in stereotypical assumptions 
Table 4.1 VP volunteers by gender

\begin{tabular}{llr}
\hline Anaesthetists & $10(6$ female $)$ & 71 \\
Obstetricians & $9(7$ female $)$ & 60 \\
Midwives & $8(8$ female $)$ & 60 \\
Nurses & $6(6$ female $)$ & 48 \\
Foundation year 2 doctors & $4(2$ female $)$ & 30 \\
Paediatricians & $3(0$ female $)$ & 33 \\
Social scientists & $2(1$ female $)$ & 24 \\
Biomedical engineers & $1(0$ female $)$ & 6 \\
General practitioners & $1(1$ female $)$ & $\mathbf{3 5 8}$ \\
Total & $\mathbf{4 4}(31$ female $)$ & \\
\hline
\end{tabular}

Source: SVP Final Report, 2014.

about their appearance and how 'young' (read inexperienced) they looked. In one case, where the author herself was present in a meeting with a representative of the Ministry of Health, the (male) turned to the obstetrician and asked her if she was a medical student. In response to a gentle assertion that she was a qualified obstetrician he replied that she looked much younger'. Another volunteer refers to the difficult relationship she had with her Ugandan line manager whom she felt did not treat her as an equal and take her seriously. Concerned about this she spoke to her predecessor (a male volunteer at the same level of seniority and discipline), 'he said the head of department was always fine with him. It sounded like it was much easier for him. I did not feel being a woman [was easy] in an environment like Uganda, even though women are able to be doctors'.

Two anaesthetic volunteers at the same level of seniority discussed the impact of gender on their relationships with local staff:

[Female volunteer] I think it's harder, harder for women.

[Male volunteer] Yep, definitely.

[Female] Without a shadow of a doubt. I think you've got to understand the society you work in, so I think being female, I wouldn't say being male is an advantage, but I would say being female is a disadvantage. Traditionally their culture in some of the tribes. You're coming into that, and then you're asking men in their fifties and sixties to listen to a young, white female (volunteer) that's a tall order in a hierarchical patriarchal society. My very first day, and I don't harp on about this, the principal [clinician] physically pushed me out of his way. Not in any forceful way, but it was definite. 
A mid-career obstetrician illustrates the inter-section of different dimensions of 'positionality' and the impact on learning and behaviour change:

Over the last week alone I must have said at least 40 times, 'don't suction the baby when it's breathing and crying', 'stop suctioning', 'please stop', 'can you put the suction down'. It's just really hard, when it's so engrained in what they do for some white person to come along and say 'no'. The working relationships I have with most of the midwives are good. They often just see me as another pair of hands, as marginally more competent than their junior doctors. They don't completely believe me in certain things but they tolerate me because they know I'll come in and do some $\mathrm{c}$ sections. The interns (doctors in training), I did have quite good relationships with. But one of them I'm not getting on with, he's being directly challenging towards me. He will challenge me on pretty much everything I say, and I'm sure it's because I'm white and female and he doesn't understand me.

Attitudes about gender are often intertwined with professional boundaries and hierarchies compounded by gender segregation; all midwives and nearly all nurses are female in Uganda. Another 'twist' on the disciplinary aspects of positionality emerges in multidisciplinary interventions. An experienced volunteer midwife explains her treatment by a local doctor during a multidisciplinary 'Well Woman' intervention:

We had a local doctor that spoke to me absolutely appallingly like, 'get this', 'do this', 'do the other'.

Professional hierarchies are by no means a feature only of Ugandan health systems. Indeed, some of the most valuable transferable skills gained by professional volunteers are connected to experiences of multidisciplinary team working. These hierarchies are often rarefied in low-resource contexts with firm boundaries obstructing effective cross-professional cooperation and team working. Another area that poses a challenge to professional volunteers concerns their treatment as 'outsiders'. We have noted above how this may stimulate active and effective learning around cultural awareness. On the other hand, it can amount to forms of quite explicit and distressing stereotyping. 


\section{Culture and 'Racial' Stereotyping}

Although translated strictly to mean 'white', the concept of 'mzungu' in Africa ${ }^{8}$ refers in more complex ways to 'wealthy' foreigners. It is also linked to an historic association of professional voluntarism with missionary-style, 'donor-recipient' voluntarism. An example of this can be seen in the nickname given to one volunteer as 'Dr Donor'. In many instances volunteers are viewed as 'cash cows' rather than co-workers. This perception of volunteer roles reinforces the expectation that volunteers come to low-resource settings as locums and donors challenging the commitment to knowledge exchange and co-presence. A good example of this is the caricature of clinicians in low-resource settings as the passive recipients of training at the opposite pole on a linear knowledge gradient; as individuals lacking capabilities and collectively, the system lacking experienced personnel. ${ }^{9}$ These myths continue to shape development interventions damaging relationships and limiting impact. They create obstacles to the achievement of the kinds of balanced professional relationships experienced in other international contexts based on collegiality.

The stereotypes associated with the 'mzungu' concept are tainted with misunderstandings about experience, skills, seniority and relative wealth. We noted above how female volunteers tend to be viewed as 'young' by senior Ugandans. In other situations, 'mzungus' are perceived by their peers as more experienced and possessing higher skills. This can place British trainees in positions where their competency is stretched (as noted above). It can also cause distress to professional volunteers.

The 'fluffy' concept of culture tends to gloss over or even excuse discriminatory and unprofessional behaviour. This narrative from an early career obstetrician shows how this stereotyping of UK volunteers can put them at risk especially when, as in this case, they are working in isolation at night:

We arrived at $8 \mathrm{pm}$. From the start midwives were sleeping in the office. The doctors worked until $3 \mathrm{am}$ when they all went to sleep and said that they would be up at $6 \mathrm{am}$. The theatre staff all stopped work at $3 \mathrm{am}$ and went to sleep, despite there being women waiting for caesareans. During this time anything could have happened. I was delivering one baby after another often on the floor in the waiting area and dealing with complex obstetric emergencies. A woman died which was emotionally draining. There was an offduty security guard taking bribes off patients and moving them to the labour ward. Then I had the doctor go mad at me for documenting in the notes 
that the reason I couldn't take a woman to theatre was because they were all asleep (despite waking them all up and informing them). He shouted at me in front of patients and staff. He showed them the notes I had written and accused me of trying to get them sacked justifying it by saying 'this is what happens in Uganda every night shift. We aren't in the UK now'. I felt vulnerable and didn't know how to get senior help. Being a mzungu in these working environments can be hard as no one really wants to help you if it interferes with their routine.

\section{Avoiding Risks through International Placements in High-Resource Settings?}

The discussion above has identified a range of potential costs, risks or 'disbenefits' associated with placing NHS employees in low-resource settings. It is important to reiterate that, from the perspectives of respondents and our experiences as volunteer managers, the gains far outweigh the costs. In practice, we have seen very few examples of these risks converting into serious outcomes. It could be argued that people do not need to travel to such extreme locations, with all the expense and general upheaval that this involves, in order to be exposed to difficult working conditions. Apart from activities that might be specifically tied to the location, the UK could arguably provide plenty of environments in which to develop the skills that are claimed to be uniquely fostered in low-income settings (McCulloch and Mishra 2009; Yule et al. 2006). However, although the UK, or any high-income country, may have some extremely deprived socio-cultural areas, anyone who has travelled or worked in low-resource settings will acknowledge that what can be experienced in such places is fundamentally different. Poverty and systemic dislocation in the UK in no way approach the levels that can be found in parts of the developing world. We asked the managers of volunteering organisations that currently provide overseas placement opportunities to comment on the difference between sending staff to high- and low-income countries. A senior coordinator with several years' experience of organising long term placements for health professionals was clear about the situation as she saw it:

I'm not absolutely sure they get that much out of going to high resource settings. I think it's completely different. I think that clinical staff may get a bit of training in comparative systems, but they'll have the same restrictions 
that affect them here. The problem in the UK is that they don't get their hands on any complex cases, like twin or breech deliveries, because of litigation and defensive practice. Because of the sheer weight of trainees, I guess, they will see so few of the cases that they need to see to progress. So when they go to a developing country they get so much more hands on. They might do observation - they might be able to observe over here, but they'll see things that they've read about in text books that over here aren't allowed to mature onto real problems - like ruptured uteruses, or diabetic foot ulcers.

Volunteers themselves who had experienced working in both high- and low-income settings highlighted similar issues. There was a general feeling that working in any foreign setting, be it low- or high-income, would be valuable and support a degree of 'systems thinking'. Even comparatively similar high-resource settings such as America or Australia, where elements such as a shared language helped to mitigate excessive culture shock, were seen as worthwhile, although it was usually acknowledged that they tended not to offer the experiential onslaught that is a feature of low-resource settings. Bethany, a recently qualified nurse who had already undertaken long-term placements in Central Africa, Tanzania and Australia, explained how the differences between the Australian and the UK high-income settings were largely administrative. The Australian healthcare system is a form of hybrid public/private arrangement which she found very easy to adjust to. It engendered none of the shock value which characterised her low-resource placements:

I think it's always good to go to somewhere different to experience something else. I think as much as it's a nice idea to stay in one job once you've qualified, I don't necessarily think it's a good idea because you don't know anything different. [In Australia] it was the same sort of culture, pretty much. Some things I'd be like, 'do you not do it like this?', and they'd say 'no' but it's just little differences. Main procedures and stuff pretty much all the same. The hospital set-ups and the hierarchies are very much the same.

A meeting with UK midwives in Australia suggested that, from a midwifery perspective, the work in Australia was less challenging than their previous work in the UK, as midwives had less autonomy in that setting. This can be contrasted with the situation described by midwives in New Zealand which has witnessed a marked policy shift in 
favour of independent midwives practicing with a very high degree of autonomy. ${ }^{10}$

Disengaging with the system one is familiar with facilitates a level of (comparative) systems-thinking that health professionals are less likely to engage in in the absence of mobility. The MOVE project did not set out to explicitly compare experiences in high- and low-resource settings and future research may prove highly valuable particularly if it is able to address experiences in areas such as the remote Australian outback. For now, we can conclude that placements in high-resource settings are less likely to present the opportunities for clinical practice or leadership that are associated with low-resource settings, and they are far more likely to act as a precursor to longer-term stays and potential settlement given the relative attractiveness of positions.

\section{SUMMARY}

This chapter has rehearsed some of the potential externality effects of international placements in low-resource settings. The most important of these concerns the financial costs of providing staff cover for NHS employees in the current human resource crisis. In addition to this, there are risks associated with such placements and it is essential that they are exposed and discussed. In practice, the perception of risk is a far greater barrier to placements than actual or inherent risks often exacerbated by forms of moral panic and lack of contextual knowledge. Risk mitigation through effective project organisation and volunteer management can significantly reduce residual risks and balance the relationship between risk management and optimal learning.

The final chapter summarises the discussion presented so far and concludes with a presentation of the Volunteer Deployment Model developed and continually refined in the course of the Sustainable Volunteering Project.

\section{Notes}

1. This is without taking into account any costs associated with the volunteer such as pensions.

2. A pilot study was completed in August 2015 and further work is planned.

3 . The SVP also provides every volunteer with 'Working in International Health' (Gedde et al. 2011). 
4. https://www.theguardian.com/world/2016/aug/18/pauline-cafferkeyebola-nurse-accused-concealing-high-temperature

5. Understanding 'Co-Presence' in the Sustainable Volunteering Project, Policy Report 2014 available at www.knowledgeforchange.org.uk/

6. The tool was designed by Drs Kim McCloud and Helen Schofield and is reproduced in Ackers, Lewis and Ackers-Johnson (2014).

7. Our sister book (Ackers-Johnson 2014) has a specific recommendation aimed at such organisations to make registration with UK Charities Commission contingent upon compliance with mainstream UK policy and practice in employment and equality matters.

8. According to Wikipedia, 'mzungu' is a Bantu language term used in the African Great Lakes region to refer to people of European descent. It is a commonly used expression among Bantu peoples in Kenya, Tanzania, Malawi, Rwanda, Burundi, Uganda, Democratic Republic of Congo and Zambia, dating back to the eighteenth century. Strictly defined as an 'aimless wanderer', the term is used in Uganda to refer generically to nonAfrican 'outsiders' - usually but not always white people.

9. A point we discuss in our sister volume (Ackers-Johnson 2014).

10. These interviews were organised as part of a scoping visit to New Zealand and a conference visit to Perth, Australia; see note 2 above.

Open Access This chapter is licensed under the terms of the Creative Commons Attribution 4.0 International License (http://creativecommons.org/licenses/ by $/ 4.0 /$ ), which permits use, sharing, adaptation, distribution and reproduction in any medium or format, as long as you give appropriate credit to the original author(s) and the source, provide a link to the Creative Commons license and indicate if changes were made.

The images or other third party material in this chapter are included in the book's Creative Commons license, unless indicated otherwise in a credit line to the material. If material is not included in the book's Creative Commons license and your intended use is not permitted by statutory regulation or exceeds the permitted use, you will need to obtain permission directly from the copyright holder.

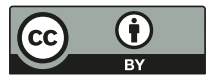

